Meta

Journal des traducteurs

Translators' Journal

\title{
Un dictionnaire multilingue de linguistique
}

\section{Anna Anastassiadis-Syméonidis}

Volume 39, numéro 4, décembre 1994

Hommage à Bernard Quemada : termes et textes

URI : https://id.erudit.org/iderudit/001885ar

DOI : https://doi.org/10.7202/001885ar

Aller au sommaire du numéro

\section{Éditeur(s)}

Les Presses de l'Université de Montréal

\section{ISSN}

0026-0452 (imprimé)

1492-1421 (numérique)

Découvrir la revue

\section{Citer cet article}

Anastassiadis-Syméonidis, A. (1994). Un dictionnaire multilingue de linguistique. Meta, 39(4), 598-614. https://doi.org/10.7202/001885ar

\section{Résumé de l'article}

Cet article comprend trois parties. Dans la première, nous présentons un bref historique de la formation du corpus. L'opération, collective dès le départ, a commencé en 1971 et continue jusqu'à ce jour. Le corpus, actuellement sur PC, contient environ 13000 articles différents, dont chacun comprend le terme grec accompagné de ses équivalents en anglais, français et allemand. La deuxième partie, consacrée au dictionnaire multilingue de linguistique, est constituée de trois sous-parties. Dans la première, après une courte exposition de quelques exemples du désordre terminologique régnant - à l'origine de cette tentative de normalisation - est présenté le public qui en sera le bénéficiaire et auquel le dictionnaire s'adresse. Dans la deuxième sous-partie, sont exposés les trois critères de sélection des vedettes, à savoir la fréquence d'usage, la répartition et les difficultés de traduction potentielles. Dans la troisième, enfin, est présentée la forme que revêtira le dictionnaire compte tenu d'un certain nombre de choix ayant trait à l'ordre alphabétique, à l'éclatement des homonymes, aux renvois, à l'indication de la catégorie grammaticale de la vedette et aux index à la fin du volume. La troisième partie comprend des réflexions nées des problèmes terminologiques auxquels nous nous sommes heurtés. Ainsi sont successivement présentés les critères d'adéquation des termes, quelques problèmes relatifs à la traduction de ceux-ci ainsi que leur forme. Dans la recherche des équivalents interlinguistiques, il est important de souligner la pérennité du signifiant malgré le changement du signifié, les correspondances interlinguistiques entre préfixes, suffixes et formants et deux cas problématiques, les faux amis et des cas de convergence et de divergence. Enfin, la forme des termes est examinée sous trois aspects, ceux de leur étymologie, des procédés déformation et de leur catégorie grammaticale.
Ce document est protégé par la loi sur le droit d'auteur. L'utilisation des services d'Érudit (y compris la reproduction) est assujettie à sa politique d'utilisation que vous pouvez consulter en ligne.

https://apropos.erudit.org/fr/usagers/politique-dutilisation/ 


\title{
UN DICTIONNAIRE MULTILINGUE DE LINGUISTIQUE*
}

\author{
ANNA ANASTASSIADIS-SYMÉONIDIS \\ Université Aristote de Thessalonique, Thessalonique, Grèce
}

\begin{abstract}
Résumé
Cet article comprend trois parties. Dans la première, nous présentons un bref historique de la formation du corpus. L'opération, collective dès le départ, a commencé en 1971 et continue jusqu'à ce jour. Le corpus, actuellement sur PC, contient environ 13000 articles différents, dont chacun comprend le terme grec accompagné de ses équivalents en anglais, français et allemand.

La deuxième partie, consacrée au dictionnaire multilingue de linguistique, est constituée de trois sous-parties. Dans la première, après une courte exposition de quelques exemples du désordre terminologique régnant - à l'origine de cette tentative de normalisation - est présenté le public qui en sera le bénéficiaire et auquel le dictionnaire s'adresse. Dans la deuxième sous-partie, sont exposés les trois critères de sélection des vedettes, à savoir la fréquence d' usage, la répartition et les difficultés de traduction potentielles. Dans la troisième, enfin, est présentée la forme que revêtira le dictionnaire compte tenu d'un certain nombre de choix ayant trait à l'ordre alphabétique, à l'éclatement des homonymes, aux renvois, à l'indication de la catégorie grammaticale de la vedette et aux index à la fin du volume.

La troisième partie comprend des réflexions nées des problèmes terminologiques auxquels nous nous sommes heurtés. Ainsi sont successivement présentés les critères d'adéquation des termes, quelques problèmes relatifs à la traduction de ceux-ci ainsi que leur forme. Dans la recherche des équivalents interlinguistiques, il est important de souligner la pérennité du signifiant malgré le changement du signifié, les correspondances interlinguistiques entre préfixes, suffixes et formants et deux cas problématiques, les faux amis et des cas de convergence et de divergence. Enfin, la forme des termes est examinée sous trois aspects, ceux de leur étymologie, des procédés de formation et de leur catégorie grammaticale.
\end{abstract}

\begin{abstract}
The article consists of three parts. In the first part a brief account of the formation of the corpus is presented, which was started in 1971 and is still in progress. The Greek Linguistic term in corpus is accompanied by its English, French and German equivalents.

The second part, devoted to the multilingual linguistic dictionary, comprises three sections. In the first one, examples of terminology disorder are presented together with the definition of the profile of its potential users. In the second section, the three criteria of item selection are given: frequency of use, distribution and potential translational difficulties. In the third section, the caracteristics of the dictionary are presented which are: alphabetical order, split of homonyms in different items, and indexes.

The third part comprises of ideas reflecting existing terminology problems. The sufficiency criteria of the terms, problems relevant to the translation of the items and the form taken, are respectively presented. Within the research of interlinguistic equivalents it is important to underline first, the durability of the signifier despite the change of the signified, second the interlinguistic correspondence between prefixes, suffixes and formants, and third, two additional problematic cases one of false friends (deceptive cognates) and the other of convergence and divergence. Finally, the form of the term is examined from three aspects: their etymology, process of formation and their grammatical category.
\end{abstract}




\section{INTRODUCTION}

Le développement impétueux de la linguistique synchronique n'a atteint la Grèce qu'en 1970. Dès lors, il s'est manifesté une prise de conscience des problèmes posés par la terminologie de cette science. On s'est vite rendu compte que, pour assurer l'enseignement efficace de la linguistique synchronique, il fallait soulever l'obstacle que représente la terminologie, partie intégrante de toute science. Il fallait, dans un champ où règne tant d'imprécision terminologique sur le plan international, arriver à résoudre un problème crucial et, qui plus est, avec le consensus le plus large.

Il a été jugé que le meilleur moyen pour y accéder était la création en premier lieu d'un vaste corpus à partir de dépouillements de textes linguistiques de nature et d'origine diverses, et, en second lieu, la traduction en grec des termes nouveaux, dans le but de confectionner un dictionnaire multilingue de terminologie linguistique.

Cet article comprend trois parties. Dans la première, nous présenterons un bref historique de la formation du corpus, dans la deuxième partie, nous examinerons l'objectif du dictionnaire multilingue de linguistique, les critères de sélection de la nomenclature ainsi que la forme du dictionnaire; enfin la troisième partie sera consacrée à quelques réflexions provoquées par les problèmes terminologiques rencontrés.

\section{LE CORPUS}

Cette entreprise, dont voici un bref historique, ne date pas d'hier. Sur l'initiative d'Aristote Katranidis', professeur à l'Université d'Illinois (USA), sous les auspices de l'Institut d'Études Néohelléniques de l'Université de Thessalonique et le financement du Ministère de la Culture et des Sciences ainsi que de la Fondation Fulbright en Grèce, ont été organisés à Thessalonique deux rencontres panhelléniques, dont le sujet était la traduction en grec de la terminologie linguistique, dans le but de la codifier sous forme de dictionnaire de terminologie linguistique.

La première rencontre a eu lieu à Thessalonique du 26 au 27 mars 1971 avec la participation de 24 chercheurs, sous la présidence de M. Setatos, professeur de linguistique à l'Université de Thessalonique, qui, après avoir fixé le but de la rencontre, a proposé une liste d'ouvrages linguistiques écrits en grec, en anglais, en français, en allemand et en russe $^{2}$, que les participants devaient dépouiller, dans le double but de recenser les termes linguistiques et de proposer par la suite pour les termes étrangers une traduction en grec. La collecte des données a été faite de manière artisanale sur des fiches, qui contenaient aussi le contexte définitoire du terme, des exemples, la source et le nom du compilateur.

La deuxième rencontre de cette commission scientifique a eu lieu de nouveau à Thessalonique du 27 au 29 décembre 1972. Le professeur M. Setatos a présenté le produit du recensement - 15000 fiches - et soulevé, dans son rapport, les problèmes posés par la traduction.

Pendant le troisième stade de ce travail, deux jeunes chercheurs, avec le soutien financier du Ministère de la Culture et des Sciences, ont travaillé pendant environ deux ans, sous la direction du professeur M. Setatos, afin de compléter le corpus (11 000 nouvelles fiches environ) et d'unifier les fiches apparentées, en présentant sur une fiche terminologique de synthèse les équivalents dans les diverses langues étrangères ainsi que les traductions proposées en grec. La forme de la fiche était la suivante : 


\begin{tabular}{|l|l|l|}
\hline terme dans une langue étrangère & exemple & traduction en grec \\
\hline anglais & & 1 \\
\hline français & & 2 \\
\hline allemand & & 3 \\
\hline italien & 4 \\
\hline russe & 5 \\
\hline définition & \\
\hline source & \\
& \multicolumn{2}{|c|}{ compilateur } \\
\hline
\end{tabular}

Un inventaire de 700 termes en anglais avec leur(s) traduction(s) en grec de $\mathrm{A}$ à $\mathrm{E}$ a été proposé aux participants - ce qui avait été convenu à la deuxième rencontre -, dans le but d'arriver à un consensus le plus large possible. Le critère qui a présidé au choix de ces termes fut le degré de leur importance en linguistique combiné avec celui que posait leur traduction en grec.

Pourtant, cette entreprise ambitieuse n'aboutit pas, pour diverses raisons, comme la suspension de l'aide financière aux deux jeunes chercheurs et l'intérêt entre temps estompé de la plupart des participants aux deux rencontres. Le fichier restait toujours à la disposition des chercheurs ${ }^{3}$, mais il lui manquait le traitement final et la mise à jour, problème qui allait s'aggravant. C'est ainsi qu'à partir de 1981, en tant qu'assistante au Département de Linguistique de l'Université de Thessalonique, j'ai commencé à compléter le fichier, p. ex. en ajoutant des termes recensés dans les dictionnaires de S. Dimitriou 4 et les index terminologiques de certains auteurs, tels que G. Guillaume, que je traduisais en grec, et à sélectionner les fiches, dans le but de confectionner un dictionnaire multilingue de terminologie linguistique. J'étais arrivée à traiter le sixième environ du fichier, mais d'autres questions plus urgentes remettaient toujours au second plan son traitement, quand le financement d'un petit projet par l'Université de Thessalonique a permis à deux de nos étudiants en maîtrise de passer sur support informatique, en utilisant DBase IIIPlus, l'ensemble du fichier, enrichi de nouveaux termes, puisés avant tout dans les 13 volumes des Actes des colloques annuels sur le grec, organisés par le Département de Linguistique de l'Université de Thessalonique depuis 1980. Ce fichier, qui ne peut pas être exhaustif, comporte actuellement 21000 articles environ, qui correspondent à peu près à 13000 articles différents. Chaque article comprend 5 champs. Les voici : terme en grec, équivalent en anglais, en français, en allemand, source.

Quel peut être l'avenir de ce fichier important? Je considère que ce fichier, qui doit rester ouvert à tous les chercheurs, doit (i) être constamment mis à jour, par le dépouillement systématique des textes fondamentaux produits en linguistique, et (ii) servir de base à tout dictionnaire de linguistique, car il faut, dès le début, envisager l'élaboration de plusieurs dictionnaires de linguistique, de différentes tailles ou de paires de langues variées, puisque l'évolution rapide dans ce domaine marquera d'obsolescence un certain nombre de vedettes et exigera l'inclusion de nouvelles unités. 


\section{LE DICTIONNAIRE}

But

En posant dès le départ comme collective la solution du problème terminologique, nous avons voulu tenter d'unifier, de standardiser la terminologie linguistique attestée en grec et d'en proposer une, là où elle manquait. Ce double effort de normalisation, qui est une réaction contre le désordre qui règne en terminologie linguistique, loin de l'immobiliser - car la linguistique est une science en plein essor -, conduira ainsi à long terme à un consensus terminologique et contribuera à l'élaboration d'un métalangage plus rigoureux.

Quelques exemples suffiront à montrer ce désordre, cette absence d'homophonie absolue à l'usage même de termes fondamentaux. Prenons les trois termes de base de F. de Saussure langage, langue, parole, et regardons leurs équivalents en grec chez quatre auteurs 5 .

$\begin{array}{llll} & \text { langage } & \text { langue } & \text { parole } \\ \text { Apostolopoulos } & \text { logos } & \text { glossa } & \text { omilia } \\ \text { Babiniotis } & \text { glossa } & \text { logos } & \text { omilia } \\ \text { Haralambopoulos } & \text { logos } & \text { glossa } & \text { omilia } \\ \text { Setatos } & \text { omilia } & \text { glossa } & \text { logos }\end{array}$

Par ailleurs, les termes langue écrite/orale sont rendus en grec par graptos/proforikos logos et acte de langage par glossiki praxi ou praxi logoy. Nous soulignerons qu'il en va ainsi d'autres termes fondamentaux en linguistique, comme aspect, connotation, idiotisme ou performance: aspect : rimatikos tropos ou opsi ou poion energeias ; connotation : syndilosi ou synypodilosi ou symparadilosi ; idiotisme : idiotismos ou idiomatismos ; performance: glossiki plirosi ou epitelesi. De même, dans quelques domaines spécialisés ou dans quelques théories peu connues, il n'y a pas de terminologie établie en grec, et tout dépend du degré de la sensibilisation de l'auteur au problème terminologique. Il est par conséquent impératif de présenter unifiée la terminologie linguistique grecque dans un ouvrage non pas normatif mais constructeur d'une norme et sélectif, dans la mesure où il est urgent d'y inclure la terminologie actuellement utilisée dans les écrits linguistiques en langue grecque.

Le moment parait donc propice pour offrir au public, qui devient de plus en plus large, une aide précieuse à court mais aussi à long terme, laquelle revêt la forme d'un dictionnaire multilingue de terminologie linguistique ${ }^{6}$. Ce dictionnaire, que nous considérons comme un instrument de consultation efficace, puisqu'il est orienté vers l'usage actuel, aidera un large public à l'encodage et au décodage des textes linguistiques. Il s'agit d'un dictionnaire conçu à l'intention des linguistes professionnels ou amateurs, des étudiants en linguistique théorique et appliquée, des enseignants de langues, des auteurs de méthodes et de manuels d'enseignement des langues, mais aussi des traducteurs et des interprètes, puisqu'il met en correspondance les termes les plus usuels en linguistique dans quatre langues. Ainsi, ce dictionnaire aidera-t-il à l'intercompréhension scientifique internationale, dans la mesure où il rendra accessible aux linguistes étrangers la littérature spécialisée écrite en grec.

\section{Sélection}

La constitution même du corpus, que nous venons d'exposer, fait que nous devions nous imposer des contraintes, car le domaine de la linguistique est particulièrement vaste et varié et la matière à traiter devient de plus en plus considérable, vu les rapports toujours nouveaux que les linguistes instaurent avec d'autres sciences. Par ailleurs, ce dictionnaire ne se veut pas exhaustif, puisque notre but est de donner des grands courants de la 
linguistique une image aussi complète que le permet le cadre de présentation en un volume. Les nombreux termes du corpus qui ne seront pas inclus dans ce dictionnaire constituent la liste d'attente pour une nouvelle édition enrichie.

Voici les critères de sélection à partir des termes imposés par le corpus, qui ont présidé à la constitution de la nomenclature: 1 . la fréquence d'usage, 2 . la répartition, 3 . les difficultés de traduction potentielles. Ainsi ont été retenus les termes les plus couramment utilisés dans le métalangage linguistique d'aujourd'hui, ce qui implique l'élimination des termes qui relèvent d'une terminologie obsolète, ésotérique ou épisodique, trop particulière à un auteur ou à un modèle et jugée être utilisée — du moins à ce jour - par un nombre restreint d'auteurs. De même n'ont pas été retenus les termes spécifiques à la description d'une langue ou d'un groupe de langues, à l'exception de ceux dont l'emploi s'est étendu à la linguistique générale, ex. sandhi et ceux qui sont utilisés à la description du grec, p. ex. crase, coronis, contracte, iotacisme.

Nous avons également été conduits à inclure les termes qui, sous le même signifiant que certains mots du vocabulaire général, expriment des notions relevant du domaine de la linguistique, ex. relation, unité, action, vérité, valeur, dépendance, car il n'est pas évident que la traduction soit la même. De même ont été retenus des termes exprimant des notions d'épistémologie générale, comme principe, exemple, interprétation, théorie, modèle, représentation, typologie, adéquat, ainsi que des termes appartenant en premier lieu à une science connexe comme la médecine, la psychologie, la sociologie ou les mathématiques, tels que aphasie, dyslexie, larynx, cordes vocales, behaviourisme, conditionnement, insécurité, stéréotype, fréquence, probabilité. Pourtant, les liens plus étroits de la linguistique avec la philologie et la grammaire traditionnelle nous ont amenés à inclure un nombre assez élevé de termes communs, tels que alphabet, syllabe, lettre, nom, verbe, adjectif, nombre, singulier, mode, indicatif, ainsi que la terminologie de la ponctuation et de la rhétorique, ex. point, virgule, hypallage, litote, oxymoron.

Enfin, les difficultés de traduction potentielles nous ont conduits à omettre les dérivés dont la relation entre le signifiant et le signifié a été jugée transparente, reposant sur une règle dérivationnelle particulièrement productive et dont le sens est compositionnel à leur structure.

On doit tout de même souligner que certains regretteront le peu de place réservée aux termes jugés très techniques appartenant en propre à tel ou tel domaine de la linguistique ou à certains développements récents de cette science. Il est évident toutefois que ce dictionnaire comportera sans doute des lacunes qui ne seront pas dues à des choix délibérés, car certaines exclusions et disproportions dans les domaines représentés sont aussi dues en partie aux limites de notre compétence.

\section{Forme}

Les considérations mêmes qui viennent d'être présentées font que la forme imposée à ce dictionnaire ne pourrait être que celle-ci :

(i) L'ordre alphabétique. Étant donné qu'un dictionnaire n'est pas lu mais consulté, l'ordre alphabétique — parfaitement arbitraire — ne pourrait qu'être adopté?.

(ii) L'éclatement des homonymes. Les termes grecs considérés comme homonymes sont présentés dans des articles séparés. Ils correspondent à des équivalents différents dans les autres langues, bien que cette différenciation quant au signifiant ne constitue pas un critère d'homonymisation, ex. arhi 1 : principe, arhi 2 : début; anaforikos 1 : relatif, anaforikos 2 : référentiel, anaforikos 3 : anaphorique (v. aussi convergence 3.2.iiib).

(iii) Les renvois. Un certain nombre de vedettes ne donnent pas lieu à des articles, mais y renvoient. Le système de renvois permet de faire l'économie dans les cas où le terme grec possède une variante, ex. domi vathoys ou vathia domi: structure profonde. 
Ce dictionnaire, ne traitant pas, de façon explicite, le sens des termes, ne présente pas de renvois concernant les relations sémantiques qui s'établissent entre eux.

(iv) Les informations de l'article. La vedette en grec sera accompagnée de sa catégorie grammaticale, que nous supposons être la même pour les autres langues, sauf si autrement indiquée. De même, le genre sera indiqué pour les substantifs grecs, français et allemands. Il a été décidé de ne pas inclure les définitions et les exemples dont on disposait dans le fichier, puisque, d'une part, le besoin impérieux était la standardisation de la terminologie et que, $d$ 'autre part, la prolifération des modèles, des théories et des écoles en linguistique exigerait un travail colossal et un temps beaucoup plus long. Enfin, dans les cas où plusieurs équivalents existent pour un terme grec, le terme d'usage le plus courant sera donné en premier.

(v) Les index. Pour rendre le dictionnaire encore plus pratique et utilisable aussi par des étrangers, nous ajouterons à la fin des index dans le sens anglais-grec, françaisgrec, allemand-grec. Dans l'avenir on pourrait envisager des dictionnaires de terminologie linguistique avec d'autres paires de langues.

\section{RÉFLEXIONS SUR LES PROBLÈMES TERMINOLOGIQUES RENCONTRÉS}

\section{Critères d'adéquation des termes}

Pour bien fonctionner dans un système terminologique, un terme doit remplir certaines conditions, qui constituent en même temps les critères de son adéquation ${ }^{8}$.

(i) Le critère d'acceptabilité. L'acceptabilité d'un terme dépend de (a) sa formation correcte, (b) son degré d'intégration dans la classe à laquelle il appartient et sa capacité de former des dérivés, ex. yfos (au lieu de styl): style, c) la circonspection, c'est-à-dire la possibilité d'éviter l'homonymie, ex. katalogos epilogis (au lieu de menoy): menu.

(ii) Le critère d'informativité. L'informativité d'un terme dépend de (a) sa dénotativité, c'est-à-dire de sa capacité de couvrir de façon adéquate ce qu'il dénote, ex. anadrasi (au lieu de feedback) : rétroaction, (b) sa transparence, c'est-à-dire du fait que la forme renseigne sur le contenu, ex. domi (au lieu de stroyktoyra) : structure, (c) sa clarté, c'està-dire de la différenciation de sa forme par rapport au signifié d'un signe déjà existant, quand il faut insister sur la différence de sens, ce qui revient à ceci : préférer la création d'un nouveau signe à la néologie sémantique, quand il y a lieu de différencier les signifiés, ex. pliroforitis (au lieu de pliroforiodotis, qui a des connotations péjoratives) : informateur.

(iii) Le critère du pouvoir d'évocation. Plus ce pouvoir est élevé, plus vite le terme est évoqué à la mémoire. Ce pouvoir est relié (a) au nombre des syllabes (la brièveté est préférable), (b) à la forme du terme (le mot est préférable au syntagme), (c) à sa cohérence (capacité de former une famille de mots), ex. morfi, morfima, allomorfa: forme/morphe, morphème, allomorphes.

(iv) Le critère de traductibilité, c'est-à-dire de la possibilité qu'a un terme traduit en grec de renvoyer au terme étranger correspondant qui lui a servi de source, ex. metashimatismos : transformation.

Pourtant, malgré les efforts multiples de théorisation de la part des linguistes, la linguistique seule n'est pas à même de prévoir dans tous les cas les conditions du succès d'un terme, car ces critères sont parfois contradictoires et s'entre-détruisent, v. p. ex. le terme proposé à (ic) qui transgresse le critère (iiib). V. aussi Quemada 1971.

\section{Problèmes de traduction}

Dès le premier stade de cette opération, pendant le recensement des termes, les chercheurs avaient à affronter des problèmes de traduction, qui se sont multipliés pendant le troisième stade de la mise en forme de la fiche terminologique de synthèse, où il fallait 
mettre en relation des termes dans six langues. Tout d'abord, à chaque fois on s'appliquait à proposer des équivalents pour les termes choisis, formés non pas suivant des règles externes au fonctionnement de la langue, comme l'assujettissement au purisme, mais suivant les règles de fonctionnement du grec. Toutefois cela n'était pas suffisant, puisqu'il fallait aussi prendre en compte la forme et le sens du terme étranger correspondant.

Outre ce qui a été dit précédemment, il me semble tout de même qu'il faudrait insister sur trois points :

(i) La pérennité du signifiant. Il est inutile de rappeler qu'en linguistique, peut-être plus qu'ailleurs, les théories évoluent ou même disparaissent, sans que cela entraîne impérativement la disparition du signifiant d'un terme. Le signifiant peut rester identique, malgré les différences profondes sur le plan du contenu, car ce qui importe avant tout c'est le signifié, la définition exacte, opératoire et univoque d'un terme dans le système terminologique où il est utilisé, $c f$. p. ex. la perpétuité du signifiant de phonème ou de morphème à travers les théories linguistiques du $\mathrm{XX}^{\mathrm{e}}$ siècle. Cela n'est pas étonnant, si on le rapproche du fait que le même signifiant, p. ex. flexion ou genre, couvre des réalités linguistiques aussi différentes en anglais, en français et en grec.

(ii) Les correspondances interlinguistiques: Bien que le critère de traductibilité évoqué ci-dessus ne fonctionne pas de façon infaillible, il est souvent possible de dégager des micro-systèmes de correspondances entre les affixes, les formants ou les constructions des langues, ce qui contribue à l'élaboration d'une terminologie avec une cohérence interne. Considérons les correspondances suivantes:

(a) Préfixes

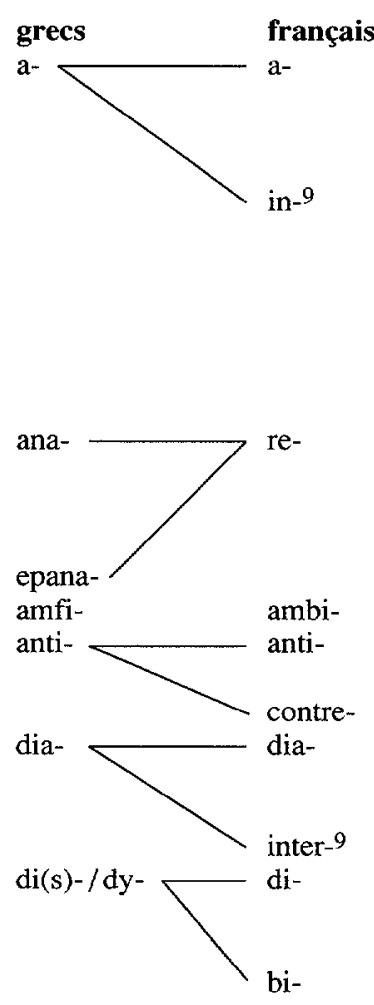

$\begin{array}{ll}\text { exemples } & \\ \text { grecs } & \text { français } \\ \text { agrammatikos } & \text { agrammatical } \\ \text { agrafia } & \text { agraphie } \\ \text { alalia } & \text { alalie } \\ \text { atonos } & \text { atone } \\ \text { apsyhos } & \text { inanimé } \\ \text { apragmatopoiito } & \text { irréel } \\ \text { aparemfato } & \text { infinitif } \\ \text { aprosopos } & \text { impersonnel } \\ \text { astathis } & \text { instable } \\ \text { anexartitos } & \text { indépendant } \\ \text { anaitiologitos } & \text { immotivé10 } \\ \text { anakatigoriopoiisi } & \text { recatégorisation } \\ \text { anasynthesi } & \text { recomposition } \\ \text { anadiplosi } & \text { redoublement } \\ \text { anadiplasiasmos } & \text { réduplication } \\ \text { epanagrafi } & \text { réćcriture } \\ \text { amfisimia } & \text { ambiguïté } \\ \text { antifrasi } & \text { antiphrase } \\ \text { antithesi } & \text { antithèse } \\ \text { antiparadeigma } & \text { contre-exemple9 } \\ \text { diasystima } & \text { diasystème } \\ \text { diahronia } & \text { diachronie } \\ \text { diathesi } & \text { diathèse } \\ \text { diaglossikos } & \text { interlinguistique } \\ \text { difthoggos } & \text { diphtongue } \\ \text { disyllavos } & \text { dissyllabe } \\ \text { digramma } & \text { digramme } \\ \text { diheilikos } & \text { bilabial } \\ & \end{array}$


dys-

meta-

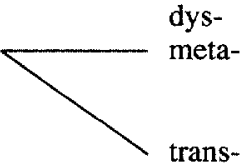

syn-

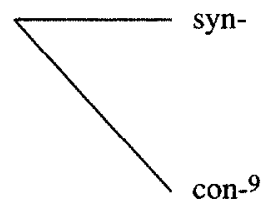

ypo-

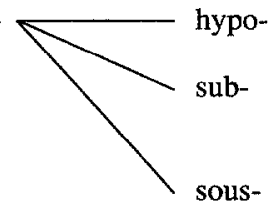

(b) Suffixes

$\begin{array}{ll}\text { grecs } & \text { français } \\ \text {-ia } & \text {-ie }\end{array}$

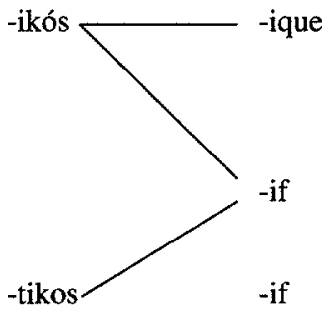

-ima

-èmel7

$\begin{array}{ll}\begin{array}{l}\text { dyadikos } \\ \text { dyslexia }\end{array} & \begin{array}{l}\text { binaire } \\ \text { dyslexie }\end{array} \\ \text { metaglossa } & \text { métalanguell } \\ \text { metafora } & \text { métaphore } \\ \text { metafonia } & \text { métaphonie } \\ \text { metashimatismos } & \text { transformation } \\ \text { metavatikos } & \text { transitif } \\ \text { metagrammatismos translittération } & 9 \\ \text { synairesi } & \text { synérèse } \\ \text { synekdohi } & \text { synecdoque } \\ \text { syntagma } & \text { syntagme } \\ \text { syllavikos } & \text { syllabique } \\ \text { synapsi } & \text { synapsie } \\ \text { symfrazomena } & \text { contexte } \\ \text { systatiko } & \text { constituant } \\ \text { syndesmos } & \text { conjonction } \\ \text { syntheto } & \text { composé } \\ \text { synanafora } & \text { coréférence12 } \\ \text { ypokoristiko } & \text { hypocoristique } \\ \text { yponymo } & \text { hyponyme } \\ \text { ypokatastasi } & \text { substitution } \\ \text { ypotaktiki } & \text { subjonctif } \\ \text { ypokeimenikos } & \text { subjectif13 } \\ \text { ypokatigoria } & \text { sous-catégorie } \\ \text { ypokodikas } & \text { sous-code } \\ \text { ypokeimenos } & \text { sous-jacent } 14\end{array}$

\begin{tabular}{ll}
\multicolumn{2}{c}{ exemples } \\
grecs & français \\
amnisia & amnésie \\
alalia & alalie \\
analogia & analogie \\
afasia & aphasie \\
kakofonia & cacophonie \\
lexikografia & lexicographie \\
analogikos & analogique \\
fonientikos & vocalique \\
fonologikos & phonologique \\
thematikos & thématique \\
simikos & sémique \\
anaforikos & relatif \\
perigrafikos & descriptif \\
geniki & génitif \\
deiktikos & démonstratif \\
elleiptikos & défectif \\
genetikos & génératif \\
eyhetiki15 & optatif \\
afairetiki & ablatif \\
ergastiki & ergatif \\
onomastiki & nominatif 16 \\
grafima & graphème \\
lexima & lexème \\
morfima & morphème
\end{tabular}




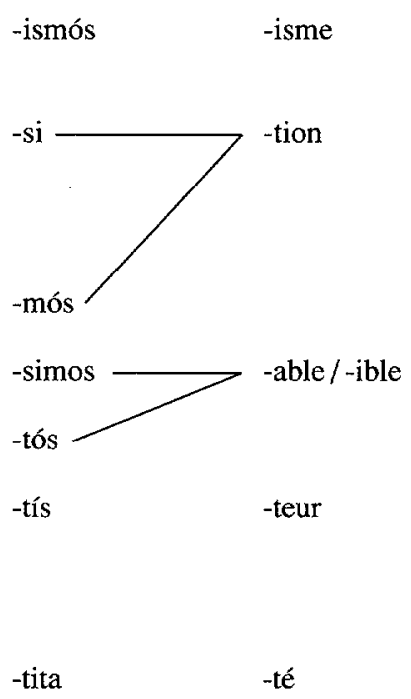

(c) Formants

$\begin{array}{ll}\begin{array}{l}\text { grecs } \\ \text { allo- } \\ \text { arhi- }\end{array} & \begin{array}{c}\text { français } \\ \text { allo- }\end{array} \\ \text { ayto- } & \text { archi- } \\ \text { imi- } & \text { hémi- } \\ \text { makro- } & \begin{array}{l}\text { semi- } \\ \text { mi- }\end{array} \\ \text { mikro- } & \text { macro- } \\ \text { mono- } & \text { non- } \\ & \text { micro- } \\ \text { mono- } & \text { uni- } \\ \text { neo- } & \text { neo- } \\ \text {-genis } & \text {-gène }\end{array}$

\begin{tabular}{|c|c|}
\hline monima & monème \\
\hline fonima & phonème \\
\hline dyadismos & binarisme \\
\hline symperiforismos & behaviourisme \\
\hline domismos & structuralisme \\
\hline arthrosi. & articulation \\
\hline afomiosi & assimilation \\
\hline elxi & attraction \\
\hline synthesi & composition \\
\hline antithesi & opposition \\
\hline periorismos & restriction \\
\hline tonismos & accentuation \\
\hline metrisimos & comptable \\
\hline diathesimos & disponible \\
\hline apodektos & acceptable \\
\hline provleptos & prévisible \\
\hline dektis & récepteur \\
\hline arthrotis & articulateur \\
\hline akroatis & auditeur \\
\hline ekfonitis & énonciateur \\
\hline omilitis & locuteur \\
\hline ihirotita & sonorité \\
\hline eikonikotita & iconicité \\
\hline omalotita & régularité \\
\hline osotita & quantité \\
\hline ammatikotita & grammaticalité \\
\hline mioyrgikotit & créativité18 \\
\hline
\end{tabular}

\section{exemples}

grecs

allofono

allomorfo

arhifonima

arhisimima

arhilexima

aytodiorthosi

aytonomos

aytonymos

imistihio

imivoithitiko

imifono

makrodomi

makroglossologia

mi empsyho

mi anthropino

mikrodomi

monosyllavos

monofthoggos

monoglossos

monopleyros

monodiastatos

neologismos

neogrammatikoi

endogenis

\section{français}

allophone

allomorphe

archiphonème

archisémème

archilexème

autocorrection

autonome

autonyme

hémistiche

semi-auxiliaire

semi-voyelle

macrostructure

macrolinguistique

non animé

non humain

microstructure

monosyllabe

monophtongue

unilingue

unilatéral

unidimensionnel

néologisme

néo-grammairiens

endogène 


\begin{tabular}{|c|c|c|c|}
\hline -grafos & -graphe & $\begin{array}{l}\text { allogenis } \\
\text { fasmatografos } \\
\text { lexikografos }\end{array}$ & $\begin{array}{l}\text { allogène } \\
\text { spectrographe } \\
\text { lexicographe }\end{array}$ \\
\hline$-\log o s$ & -logue & dialektologos & dialectologue \\
\hline -onym(i)a & -onym(i)e & $\begin{array}{l}\text { metonymia } \\
\text { toponymia }\end{array}$ & $\begin{array}{l}\text { métonymie } \\
\text { toponyme }\end{array}$ \\
\hline & & omonymo & homonyme \\
\hline -fonos & -phone & gallofonos & francophone \\
\hline tayto- & tauto- & taytologia & tautologie \\
\hline -poio & -iser $/$-ifier 19 & lexikopoio & lexicaliser \\
\hline & & $\begin{array}{l}\text { pathitikopoio } \\
\text { katigoriopoio }\end{array}$ & $\begin{array}{l}\text { passiviser } \\
\text { catégoriser }\end{array}$ \\
\hline & & oydeteropoio & neutraliser \\
\hline & & posotikopoio & quantifier \\
\hline & & tropopoio & modifier \\
\hline & & 80 & Isom \\
\hline
\end{tabular}

La présentation de ces éléments, bien qu'elle ne soit pas exhaustive, permet d'établir des correspondances entre le grec et le français, qui sont d'un grand secours pour le traducteur.

(d) Constructions

\begin{tabular}{llll} 
grecs & français & \multicolumn{2}{c}{ exemples } \\
AN & NA & $\begin{array}{l}\text { grecs } \\
\text { graptos logos } \\
\text { dipli arthrosi } \\
\text { glossikos atlantas } \\
\text { fonitikes hordes } \\
\text { eythis logos }\end{array}$ & $\begin{array}{l}\text { langue écrite } \\
\text { double articulation } \\
\text { atlas linguistique } \\
\text { cordes vocales } \\
\text { discours direct }\end{array}$ \\
& & ameso systatiko & constituant immédiat \\
& & praxi logoy & acte de parole \\
& & simio arthrosis & point d'articulation \\
& pedio diasporas & champ de dispersion \\
glossiko epipedo & niveau de langue \\
mitera glossa & langue-mère
\end{tabular}

Mais ces correspondances ne sont pas sans exception, ex. les termes phrase noyau (NN) et phrase nucléaire (NA) sont rendus en grec par piriniki protasi (AN), structure profonde (NA) est rendu par vathia domi (AN) mais aussi par domi vathoys (NNgén.).

(iii) Cas problématiques. Ces correspondances une fois établies, on comprend aisément que tout ne soit pas résolu pour le traducteur. Il reste à examiner deux cas de figure qui posent problème.

(a) Les faux-amis. Le problème repose sur le fait que le signifiant d'un terme se rapproche du signifiant d'un terme dans une autre langue, sans pour autant qu'il y ait la correspondance attendue sur le plan du contenu, p. ex. en grec epitheto: adjectif, terme désignant une catégorie grammaticale, se rapproche du français épithète, qui désigne une fonction et qui correspond au grec epithetikos prosdiorismos. De même, le terme frasi correspond, dans une de ses acceptions, au terme anglais phrase et au français syntagme, mais, dans une autre acception, il correspond au français phrase et a alors protasi comme synonyme en grec. Nous pourrions dire que les faux-amis sont des paronymes interlinguistiques et qu'ils se caractérisent par des différences d'extension, malgré leur origine commune. Toutefois, comme dans les paires de faux-amis il y a souvent un troisième 
terme qui intervient dans une des deux langues ( $c f$. le cas de frasi), nous allons examiner ces cas sous la rubrique convergence et divergence, qui sont dues, elles aussi, à des écarts d'extension entre les termes, à la non-superposition de leur aire sémantique.

(b) Convergence-Divergence. La convergence et, son complémentaire, la divergence sont les deux facettes de la bifurcation qui se présente «[1]orsque la traduction d'un mot oblige à choisir entre deux ou plusieurs équivalents» (Darbelnet 1970: 94). Cela admis, c'est l'orientation de la bifurcation en fonction de la langue maternelle des usagers qui jugera s'il s'agit d'un cas de convergence ou de divergence. Il y aura convergence lorsque la différence notée entre phrase, syntagme et frasi est préjudiaciable au grécophone. Dans le cas contraire, il y aura divergence. Les cas de convergence sont de loin les plus fréquents et cela est dû, dans la grande majorité des cas, au fait que le français ainsi que l'anglais puisent leurs lexèmes soit dans le fonds latin soit dans le fonds grec ancien. Par contre, le grec moderne puise presque exclusivement dans le fonds grec ancien, ou, dans la plupart des cas, emprunte directement au français ou à l'anglais, qui ont, eux, préalablement puisé dans le fonds grec ancien. Les emprunts directs d'origine romane que fait le grec moderne dans la terminologie linguistique sont extrêmement rares, ex. norma $<\mathrm{fr}$. norme, apriorismos < fr. apriorisme, stroyktoyralismos < fr. structuralisme, mais qui est dernièrement supplanté par le calque domismos. Le grec se trouvant ainsi privé d'une source d'emprunt, le traducteur doit recourir à d'autres moyens pour combler cette lacune, ex. accent : tonos, ton : moysikos tonos, bilinguisme : diglossia et diglossie : diploglossia ou dimorfia. Exemples de convergence:

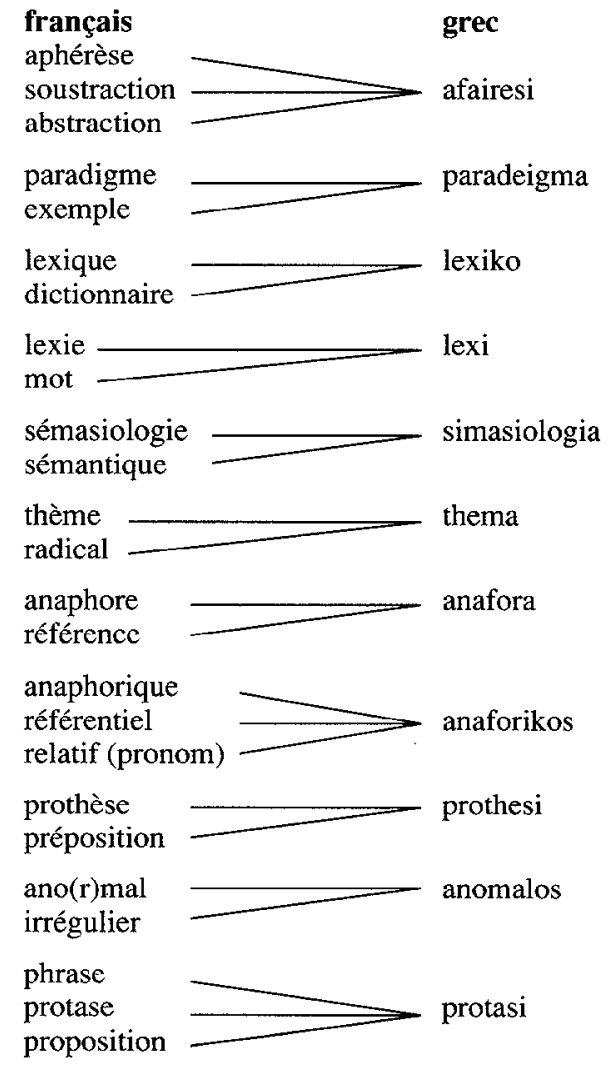




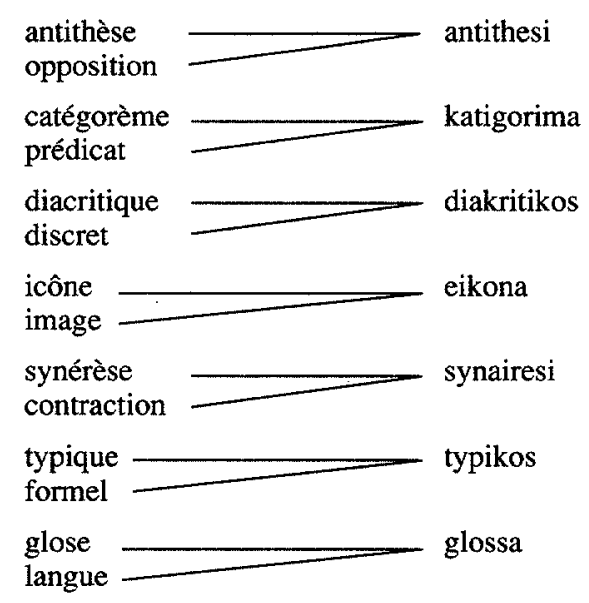

Néanmoins, comme nous venons de dire, il y a quelques cas de convergence où aucun des termes impliqués n'est d'origine grecque, ex.:

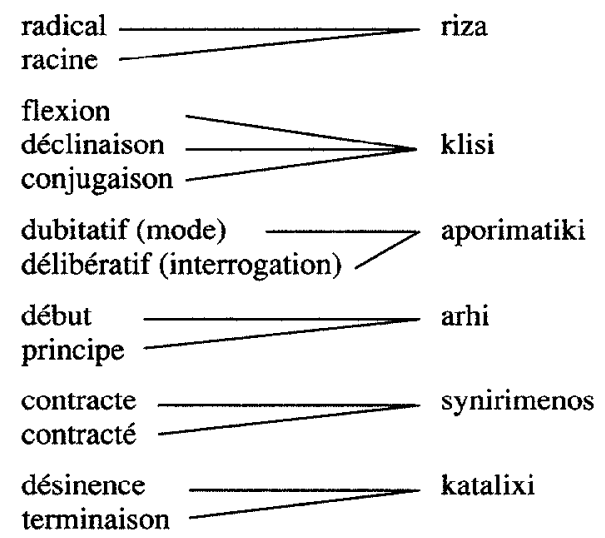

Il n'est sans doute pas inutile de souligner ici que la convergence ne se limite pas aux seuls lexèmes, mais qu'elle touche également les affixes et les formants. Aux nombreux exemples déjà présentés (ex. sub-/ hypo-/ sous-: ypo-), ajoutons-en encore deux :

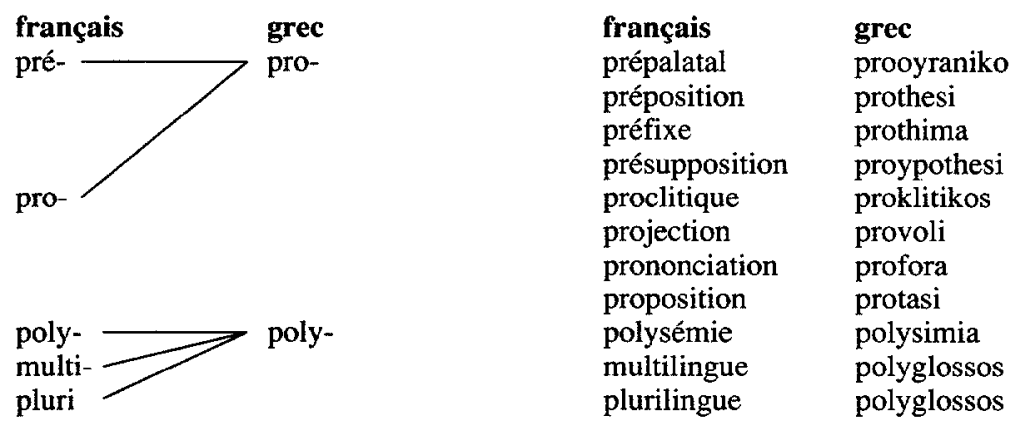

Considérons maintenant quelques exemples de divergence: 


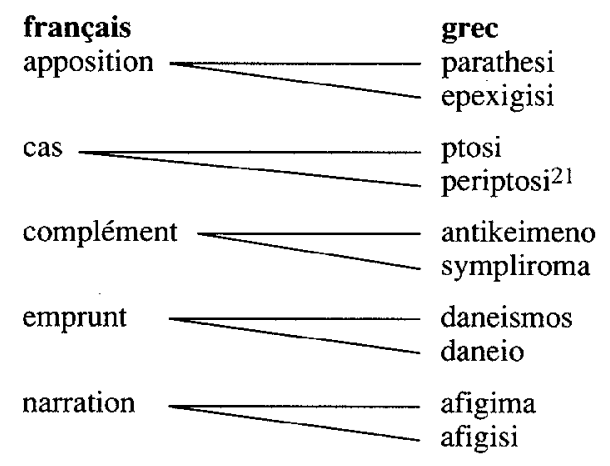

\section{Forme des termes}

Une situation nouvelle est en train de se créer en linguistique grecque avec l'essor de la linguistique synchronique, qui, comme toute science nouvelle ou en voie d'évolution, a besoin d'une terminologie adaptée à son objet. Pour répondre à ce besoin, nous avons eu recours aux multiples procédés de néologie. Nous allons examiner la néologie terminologique ${ }^{22}$ sous trois aspects : l'étymologie, un aspect diachronique, et deux aspects synchroniques, les procédés de formation des termes et la catégorie grammaticale à laquelle ils appartiennent.

(i) L'étymologie. La plus grande partie de la terminologie linguistique grecque a été construite à partir de lexèmes d'origine grecque. Ces lexèmes sont (i) soit légués de la tradition grecque, comme de nombreux termes de la rhétorique, (ii) soit empruntés à une langue étrangère qui les avait empruntés elle aussi au grec ancien, dans le but de les utiliser comme éléments de dérivés ou de composés. Dans ce cas-ci, le grec moderne ne recrée pas le terme, mais se limite généralement à translittérer le néologisme et à l'intégrer dans sa catégorie grammaticale, p. ex. syntagmatique: syntagmatikos ${ }^{23}$. (iii) Soit enfin ces lexèmes participent à la formation de calques, où la fidélité de la traduction est variable, ex. structuralisme: domismos. Nous considérons que notre remarque que le français peut servir de maillon pendant l'opération de la création d'un calque en grec n'est pas dénuée d'intérêt. En effet, le terme grec qui est le correspondant d'un terme anglais présente une forme qui est calquée sur celle de l'équivalent en français, ex. angl. feedback : fr. rétroaction: grec anadrasi (v. aussi Anastassiadis-Syméonidis 1994 : 143).

Une minime partie de cette terminologie comprend des emprunts directs à quelques langues, mais il est important de souligner que ces termes sont utilisés tels quels par un grand nombre de langues. Considérons les exemples suivants:

$\begin{array}{lll}\text { anglais } & \text { français } & \text { grec } \\ \text { bit } & \text { bit } & \text { bit } \\ \text { calque } & \text { calque } & \text { calque /metafrastiko daneio } 24 \\ \text { click } & \text { click/clic } & \text { klik } \\ \text { cliché } & \text { cliché } & \text { klise } \\ \text { corpus } & \text { corpus } & \text { corpus } \\ \text { pidgin } & \text { pidgin } & \text { pidgin } \\ \text { sabir } & \text { sabir } & \text { sabir } \\ \text { sandhi } & \text { sandhi } & \text { sandhi } \\ \text { satem } & \text { satem } & \text { satem } \\ \text { schwa } & \text { schwa/chva } & \text { schwa } \\ \text { slang } & \text { argot }(m .) & \text { slang/argko (f.) } \\ \text { standard } & \text { standard } & \text { stantar } \\ \text { pitch } & \text { pitch } & \text { pitch }\end{array}$




$\begin{array}{lll}\text { behavio(u)rism } & \text { behavio(u)risme } & \text { bihaviorismos } \\ \text { concordance } & \text { concordance } & \text { kogkorntans / kogkorntantsia } \\ \text { glide } & \text { glide } & \text { glide } \\ \text { hapax } & \text { hapax } & \text { apax } \\ \text { index } & \text { index } & \text { index }\end{array}$

Voici des exemples de termes linguistiques en grec et en français fondés sur des lexèmes empruntés au grec ancien directement ou au moyen du latin :

\begin{tabular}{ll} 
grec & français \\
akoystikos & acoustique \\
alligoria & allégorie \\
allofono & allophone \\
alfavitikos & alphabétique \\
amnisia & amnésie \\
analogia & analogie \\
analytikos & analytique \\
anthropologia & anthropologie \\
aoristos & aoriste \\
arhaismos & archaisme \\
katahrisi & catachrèse \\
diahronia & diachronie \\
dialektos (f.) & dialecte (m.) \\
ikonomia & économie \\
elleipsi & ellipse \\
emfasi & emphase \\
egklisi & enclise \\
ethnikos & ethnique \\
etymologia & étymologie \\
eyfimismos & euphémisme \\
gnomikos & gnomique \\
aplologia & haplologie \\
armonia & harmonie \\
omoglossos & homoglosse \\
omonymo & homonyme \\
ideogramma & idéogramme \\
isomorfismos & isomorphisme \\
lexikografia & lexicographie \\
morfologia & morphologie \\
neologia & néologie \\
onomasiologia & onomasiologie \\
onomatopoiia & onomatopée \\
orthografia & orthographe \\
fatikos & phatique \\
fonologia & phonologie \\
paragrafos & paragraphe \\
parafrasi & paraphrase \\
perifrasi & périphrase \\
polysimia & polysémie \\
prosodia & prosodie \\
rythmos & rythme \\
syllavi & syllabe \\
& \\
\hline
\end{tabular}




$\begin{array}{ll}\text { symvolo } & \text { symbole } \\ \text { syntaxi } & \text { syntaxe } \\ \text { systima } & \text { système } \\ \text { taxinomikos } & \text { taxinomique } \\ \text { typologia } & \text { typologie } \\ \text { vasi } & \text { base }\end{array}$

(ii) Les procédés de formation des termes. Pour voir quelles formes revêt la néologie terminologique en linguistique, reprenons dans ses grands traits notre typologie des néologismes (Anastassiadis-Syméonidis 1986:130), fondée sur un système d'oppositions binaires de nature morphophonologique et syntaxique. Nous observons que la terminologie linguistique se construit de la même façon. La base de cette typologie est l'unité lexicale et ses deux faces, le signifiant et le signifié. La création peut concerner soit les deux faces à la fois soit l'une seulement. Dans le premier cas, il en résulte un nouveau signe, ex. fonima : phonème, dans le deuxième cas, en terminologie linguistique, un nouveau sens se présente sous un signifiant déjà existant en grec, c'est-à-dire qu'il s'agit soit (i) du néologisme sémantique, ex. paradeigma: exemple devient aussi l'équivalent de paradigme, sthenos: force // (chimie) valence, devient aussi l'équivalent de valence (ling.) ${ }^{25}$, soit (ii) de la conversion, c'est-à-dire de la création d'un terme dont le signifiant existait déjà mais appartenait à une autre catégorie grammaticale, ex. eyhetiki $(\mathbf{f}$.$) : optatif (\mathrm{m}$.$) ,$ oyraniko (neutre): palatale (f.), galliki (f.) ou gallika (neutre): français (m.) (adjectifs substantivés).

Par ailleurs, le terme nouveau peut être égal à un mot graphique, ex. psyhoglossologia: psycholinguistique, morfima: morphème, lexikopoio: lexicaliser, ou en dépasser les limites et prendre la forme d'un syntagme lexical, ex. syntagmatikos axonas : axe syntagmatique, elahisto zeygos: paire minimale, glossiki ikanotita: compétence linguistique, timi alitheias : valeur de vérité, kanones epanagrafis : règles de réécriture ${ }^{26}$.

Enfin le terme ou l'élément du terme qui se présente sous la forme de mot graphique, du point de vue morphologique, peut constituer une unité simple, ex. domi: structure, dentro: arbre, une unité dérivée à l'aide d'un affixe, ex. fonima : phonème, metaglossa : métalangue, ou une unité composée, ex. allofono: allophone, monoglossos: unilingue.

Cependant, certains termes présentent une fluctuation concernant leur signifiant, ce qui n'est pas rare en néologie, même scientifique. Cette fluctuation peut être due à l'emploi soit d'éléments concurrents, p. ex. heilosi ou heilopoiisi : labialisation, soit de variantes affixales, ex. protasikos ou protasiakos : propositionnel, soit de constructions concurrentes, ex. domi epifanias (NNgén.) ou epifaniaki domi (AN): structure de surface, epilektikos periorismos (AN) ou periorismos epilogis (NNgen) : contrainte de sélection. Ces fluctuations peuvent prendre également la forme de synonymes sans relations morphologiques, chose encore plus grave, ex. onoma ou oysiastiko: nom ou substantif. Je considère qu'en néologie terminologique il est tout à fait possible d'espérer l'élimination des fluctuations, qui ne font que nourrir un sentiment d'insécurité même parmi les linguistes professionnels.

(iii) La catégorie grammaticale des termes. II n'est pas étonnant que, dans le corpus, la classe la plus privilégiée soit celle du nom, qui comprend également les syntagmes lexicaux dont la tête est un nom. Cela est dû à la nature même du nom en tant que signe qui dénomme par excellence. Il faut également prendre en compte la nominalisation, le procédé de formation de noms sur une base verbale, ex. proypothesi: présupposition, arthrosi : articulation, ou adjectivale, ex. grammatikotita: grammaticalité, dyadismos: binarisme.

Pour ce qui concerne la classe adjectivale, la terminologie linguistique grecque comprend des adjectifs simples, ex. aplos : simple, eythis: direct, amesos: immédiat, 
ainsi que des adjectifs dérivés, produits d'une adjectivation sur une base verbale, ex. elleiptikos: défectif, ergastiki : ergatif, apodektos : acceptable ou nominale, ex. fonientikos : vocalique, simikos: sémique. Le corpus comprend aussi un nombre réduit de verbes, qui se présentent le plus souvent à la troisième personne et à la voix médio-passive, ex. proferetai: il se prononce, eisagetai : il est introduit, tonizetai : il est accentué. Il est sans doute exact - ce que beaucoup d'autres avant nous ont constaté — que «la langue scientifique est caractérisée par la réduction des formes verbales au profit des formes nominales et adjectivales» (Kocourek 1982: 70).

En conclusion, je voudrais insister sur deux points. Cette opération, qui a demandé beaucoup de temps et d'efforts, ne doit pas être considérée comme une affaire close. Le corpus doit être continuellement mis à jour et les propositions de traduction pour les termes nouveaux toujours occuper notre esprit. Toujours est-il qu'il reste encore des problèmes à résoudre et des suggestions à faire.

Enfin, il me semble que les études sur les langues romanes, qui foisonnent ces derniers temps, doivent aussi comprendre le grec moderne, qui, bien qu'il n'y appartienne pas génétiquement, comporte un grand nombre de traits communs avec elles, qui sont dus non seulement à la source indo-européenne commune, mais aussi et surtout aux influences réciproques millénaires.

Notes

* Je remercie E. Kougioumtzoglou-Roucher pour sa lecture attentive du manuscrit.

1. Mort prématurément en 1973.

2. En tout 32 livres en grec et 35 en langue étrangère, p. ex. parmi les dictionnaires spécialisés, le Dictionnaire de terminologie linguistique d'Ahmanova, le Dictionnaire multilingue de Nash, le Lexique de la terminologie linguistique de Marouzeau, parmi les ouvrages collectifs Le Langage sous la direction d'A. Martinet, et, parmi les textes de linguistique, la Sémantique structurale de Greimas, les Éléments de linguistique générale de Martinet, les Essais de linguistique générale de Jakobson, Aspects of the Theory of Syntax de Chomsky.

3. Par exemple, pour la traduction en grec des termes linguistiques anglais et français, le Bureau de termes scientifiques et de néologismes de l'Académie d'Athènes avait consulté ce fichier (Bulletin 1988:45-48, 88-89).

4. Il s'agit de dictionnaires qui présentent des équivalents dans quatre langues et analysent également le contenu, mais leur qualité n'est pas très bonne.

5. Les exemples sont puisés dans Motsiou (1985: 298). Les termes grecs ont été translittérés. Tout de même la fricative interdentale non voisée est rendue par th, le éta par $i$ et l'oméga par $o$. L'accent lexical n'est noté que pour les suffixes. Le premier de ces auteurs est le traducteur en grec du Cours de linguistique générale de F. de Saussure, et les trois autres sont des professeurs de linguistique.

6. Date de parution: 1995 . Nombre de vedettes : environ 6000.

7. Le groupement sémantique adopté dans certains dictionnaires de linguistique n'est généralement pas utilisé dans les dictionnaires multilingues, mais plutôt dans les ouvrages revêtant la forme de dictionnaires encyclopédiques.

8. Toute cette partie est fondée sur le texte de Babiniotis 1993.

9. En français $a-$, anti-, dia-, di(s)-/dy, méta- et syn-se placent devant un lexème d'origine grecque, tandis que in-, contre-, inter-, bi-, trans- et con-se placent respectivement devant un lexème d'origine latine.

10. Pourtant in- correspond parfois en grec à en-, ex. infixe: enthima, inhérent: eggenis, intensif: entatikos, indirect : emmesos, indice : endeiktis.

11. Toutefois dans métalangue, nous avons la combinaison d'un élément grec meta et d'un élément d'origine latine langue; il s'agit des hybrides, condamnés par les puristes.

12. Pourtant synomilitis : interlocuteur $\left({ }^{*}\right.$ collocuteur $)$.

13. Mais suffixe : epithima (*ypothima).

14. La distribution des éléments français est la suivante : sub- devant les lexèmes d'origine latine, hypo- devant ceux d'origine grecque, sous- devant des mots libérables.

15. La forme -iki du féminin s'explique par le fait qu'il s'agit d'un adjectif substantivé au féminin, puisque les noms des modes verbaux sont du féminin comme, d'ailleurs, le mot egklisi 'mode' lui-même. De même les noms des cas, qui forment un microsystème en français (noms en -if) et en grec (noms en -iki). Le mot ptosi 'cas' est féminin en grec.

16. Mais organiki : instrumental. 
17. Il s'agit d'un suffixe spécial à la terminologie linguistique, qui n'est pas répertorié dans les listes des suffixes du français ni du grec. Contrairement aux autres suffixes, il possède un sens lexical 'unité du niveau de la langue', ce qui est dû à son statut de suffixe appartenant en propre à une terminologie.

18. De même, la dérivation d'un nom sur une base adjectivale en -tós t-able ou -ible donne respectivement un nom en -tótita/-abilité ou -ibilité, ex. apodektotita : acceptabilité, analysimotita : analysabilité, anagnosimotita : lisibilité.

19. La classification étant faite à partir du grec, -poio est classé parmi les formants, tandis que les équivalents en français ont le statut de suffixes.

20. De même le substantif dérivé sur la base d'un verbe en -poio/-iser ou -ifier se formera avec le suffixe -poiisi -isation ou -ification respectivement, ex. nominalisation: onomatopoiisi, lexicalisation: lexikopoiisi.

21. Ne s'agissant pas d'un terme, mais d'un mot du vocabulaire général, les cas d'ambiguïté sont très limités.

22. Rondeau (1981: 121-122) utilise le terme de néonymie, pour désigner la néologie en langue de spécialité

23. Mais onomastique : onomatologia (*onomastiki pour éviter sans doute l'homonymie avec onomastiki : nominatif).

24. Calque de calque.

25. Le nouveau sens peut être emprunté directement à une langue étrangère ou indirectement au moyen d'un vocabulaire scientifique de la même langue, ex. sthenos : valence.

26. Parfois ces syntagmes lexicaux, à l'intérieur d'une théorie ou d'un modèle linguistique prennent en langue étrangère la forme d'un sigle, ex. TGG (Transformational Generative Grammar), LFG (Lexical Functional Grammar), DP (Deep Structure), SS (Surface Structure). Ce n'est pas le cas en grec, du moins en ce qui concerne la langue orale.

\section{RÉFÉRENCES}

ANASTASSIADIS-SYMÉONIDIS, A. (1986) : La néologie en grec moderne, Bulletin Scientifique de la Faculté des lettres de l'Université de Thessalonique, $\mathrm{n}^{\circ} 65$.

ANASTASSIADIS-SYMÉONIDIS, A. (1994): Emprunt néologique en grec moderne; emprunts directs du grec au français et à l' anglo-américain - analyse morphophonologique, Thessalonique.

BABINIOTIS, G. (1993) : «Le côté linguistique des termes scientifiques», To Vima, 20 juin.

Bulletin de terminologie scientifique et de néologismes (1988) : fasc.1, Académie d'Athènes - Bureau de termes scientifiques et de néologismes, Athènes.

DARBELNET, J. (1970) : «Dictionnaires bilingues et lexicologie différentielle», Langages, 19, pp. 92-102.

DIMITRIOU, S. (1980) : Dictionnaire de termes de communication et d' analyse sémiotique, Athènes, Kastaniotis.

DIMITRIOU, S. (1983) : Dictionnaire de termes de linguistique, vol. A' et $\mathrm{B}^{\prime}$, Athènes, Kastaniotis.

KOCOUREK, R. (1982) : La langue française de la technique et de la science, Wiesbaden, Oscar Brandstetter

MEILLET, A. (1961): «Sur la terminologie de la morphologie générale», Linguistique historique et linguistique générale, t. 2, Klincksieck, pp. 23-35.

MOTSIOU, V. (1985): «Sur la standardisation de la terminologie linguistique grecque», Studies in Greek Linguistics - Proceedings of the 3rd Annual Meeting of the Department of Linguistics, Faculty of Philosophy, Aristotelian University of Thessaloniki, 26-28 April 1982, pp. 295-305.

QUEMADA, B. (1971) : «À propos de la néologie - Essai de délimitation des objectifs et des moyens d'action», La Banque des mots, 2 , pp. 137-150.

RONDEAU, G. (1981): Introduction à la terminologie, Montréal. 